\title{
A INTERAÇÃO EXISTENTE EM EQUIPES QUE ATUAM NA ESTRATÉGIA SAÚDE DA FAMÍLIA E AS FAMÍLIAS POR ELAS ATENDIDAS
}

Suzanne Pires de Sousa ${ }^{1}$, Jeane Almeida de Araújo² ${ }^{2}$ Liliane da Consolação Campos Ribeiro ${ }^{3}$, Luciana Fontana Monteiro Mirtes Ribeiro ${ }^{5}$, Thamara de Souza Campos ${ }^{6}$

\begin{abstract}
RESUMO: Uma das propostas da Estratégia Saúde da Família é promover assistência integral às famílias, de forma que haja uma interação adequada entre os profissionais de saúde e as pessoas no nível individual, familiar e coletivo. Este estudo tem como objetivo identificar o tipo de interação existente entre esses profissionais e as famílias atendidas nas Unidades de Saúde da Família de um município no interior de Minas Gerais. Os dados foram obtidos por meio de entrevistas estruturadas, gravadas para posterior análise baseada no método de análise de conteúdo. Os resultados foram divididos em quatro formas de interação: visitas domiciliares, consultas, comunicação e trabalho dos agentes comunitários de saúde. Com este trabalho, conclui-se que a interação entre profissionais de saúde e famílias contribui para o trabalho da ESF.
\end{abstract}

PALAVRAS-CHAVE: Relações profissional-família; Saúde da família; Família.

\section{THE INTERACTION BETWEEN TEAMS THAT ACT IN FAMILY HEALTH STRATEGY AND THE FAMILIES ASSISTED BY THEM}

\begin{abstract}
One of the proposals of the Family Health Strategy (FHS) is to promote comprehensive care for families, so that there is an adequate interaction between health professionals and people in the individual, family and collective aspects. This study aims to identify the type of interaction between these professionals and families treated at the Family Health Unity (FHU) in a county of the state of Minas Gerais. The study was submitted and approved by the Ethics Committee of the Federal University of the Jequitinhonha and Mucuri. Data were collected through structured interviews recorded for later analysis based on the method of content analysis. The results were divided into four ways of interaction: home visits, consultations, talk and work of community health workers. This work concluded that the interaction between health professionals and families contributes to the work of the FHS.
\end{abstract}

KEYWORDS: Professional-family relations; Family health; Family.

\section{LA INTERACCIÓN EXISTENTE EN EQUIPOS QUE ACTÚAN EN LA ESTRATEGIADE SALUD DE LA FAMILIAY LAS FAMILIAS ATENDIDAS POR ELLOS}

RESUMEN: Una de las propuestas de la Estrategia de Salud de la Familia (ESF) es promover asistencia integral a las familias, de modo a que existe una interacción adecuada entre los profesionales de salud y las personas en nivel individual, familiar y colectivo. Este estudio tiene como objetivo identificar el tipo de interacción entre estos profesionales y las familias tratadas en la Salud de la Familia (USF) de un municipio en el interior de Minas Gerais. Los datos fueron recolectados a través de entrevistas estructuradas, grabadas para posterior análisis basado en el método de análisis de contenido. Los resultados fueron divididos en cuatro formas de interacción: visitas a domicilio, consultas, comunicación y trabajo de los agentes comunitarios de salud. Con este trabajo, conclúyese que la interacción entre profesionales de salud y familias contribuye para el trabajo de la ESF.

PALABRAS CLAVE: Relaciones profesional-familia; Salud de la familia; Familia.

\footnotetext{
${ }^{1}$ Aluna do Curso de Graduação em Enfermagem da Universidade Federal dos Vales do Jequetinhonha e Mucuri-UFVJM.

${ }^{2}$ Aluna do Curso de Graduação em Enfermagem da Universidade Federal dos Vales do Jequetinhonha e Mucuri-UFVJM.

${ }^{3}$ Enfermeira. Mestranda em Ciências da Saúde pela Universidade Federal de Minas Gerais-UFMG. Especialista em Saúde Pública para a Educação e em Saúde da Família pela UFMG. Docente do Departamento de Enfermagem da UFMG.

${ }^{4}$ Aluna do Curso de Graduação em Enfermagem da Universidade Federal dos Vales do Jequetinhonha e Mucuri-UFVJM.

${ }^{5}$ Meste em Ciências da Saúde pela Faculdade de Medicina da UFMG. Especialista em Saúde Pública e em Saúde da Família. Docente do Departamento de Enfermagem da UFVJM.

${ }^{6}$ Aluna do Curso de Graduação em Enfermagem da Universidade Federal dos Vales do Jequetinhonha e Mucuri-UFVJM.
}

Autor correspondente:

Liliane da Consolação Campos Ribeiro

Universidade Federal de Minas Gerais

Rua da Glória, 187 - 39100-000 - Diamantina-MG, Brasil

Recebido: 24/05/09

E-mail: lilianeribeiro@hotmail.com

Aprovado: 27/09/09 


\section{INTRODUÇÃO}

Família é a união de pessoas que escolhem conviver por afetividade e assumem compromisso de cuidado mútuo, muitas vezes atua como norteadora na formação de seus membros, tendo importância como reflexo das várias atitudes a serem tomadas ${ }^{(1)}$. Além disso, age como importante apoio para cada um de seus componentes. Um estudo mostra que, em todos os aspectos da vida, "o indivíduo aprende muito observando e copiando modelos e atitudes, do que pelas informações tiradas de livros e manuais, ou recebidas por meio de textos cientificamente corretos"(2:143).

No contexto de amparar cada grupo familiar, está incluído o profissional de saúde, que atua de forma a se importar com as observações das questões históricas e sociais da família, o qual interfere na formação e no cumprimento de sua função social, pois a análise detalhada desses fatores o auxiliará na intervenção do processo saúde-doença. A inserção desse profissional não é necessariamente atuar em caráter curativo, abrange muito além desse tipo de intervenção, com o intuito de ajudar na manutenção e na melhora da saúde familiar.

No Brasil, o Sistema Único de Saúde-SUS configura as Unidades Básicas de Saúde-UBS como a principal porta de entrada para a assistência à saúde. Entre essas, as Unidades de Saúde da Família-USF encontram papel de destaque, tendo como perspectiva resolver a maioria dos problemas de saúde da população sob sua área de abrangência. Além disso cabe a ela a reorganização da Atenção Básica à Saúde-ABS, por meio de um conjunto de ações de saúde individuais e coletivas que abrangem promoção e proteção da saúde, prevenção de agravos, tratamento e reabilitação do cliente"(3:449).

A Estratégia de Saúde da Família-ESF criada em 1994, como Programa Saúde da Família-PSF fixase na busca do atendimento pleno da família, propiciando o enfrentamento e a tentativa de resolução de problemas identificados, por meio de integração de sua equipe com a população. Essa equipe é composta por um médico, um enfermeiro, um auxiliar ou técnico de enfermagem, um cirurgião dentista, um técnico e/ ou um atendente de higiene dental e até dez agentes comunitários de saúde-ACS. Podem ser incluídos também outros profissionais de nível superior, por meio do Núcleo de Assistência a Saúde da Família-NASF(3).

A ESF tem como objetivos organizar a demanda espontânea, trabalhar com agendamento e busca ativa, dar ênfase às necessidades individuais e familiares de sua população adscrita. Para isso, "são previstas ações de tratamento, prevenção e promoção à saúde, por meio de práticas educativas participativas, inclusive no próprio domicílio”(4:25).

Uma abordagem interessante é feita por alguns autores $^{(5: 66)}$, quando afirmam que na ESF

\begin{abstract}
a família deve ser atendida de forma integral e em seu espaço social, ou seja, a pessoa deve ser abordada em seu contexto socioeconômico e cultural, e reconhecida como sujeito social portadora de autonomia, reconhecendo que é na família que ocorrem interações e conflitos que influenciam diretamente na saúde das pessoas.
\end{abstract}

Além disso, a família poderá atuar de modo a ajudar os profissionais de saúde, uma vez que fornecem informações necessárias e atuam no cuidado direto de seus integrantes. Assim, os profissionais devem apresentar atitude diferenciada, pautada no respeito, na ética e no compromisso com as famílias pelas quais são responsáveis, mediante a criação de vínculos de confiança e de afeto. A partir desse momento, haverá atuação de forma participativa na construção de ambientes mais saudáveis no espaço familiar ${ }^{(6)}$.

Os processos de produção de saúde dizem respeito, necessariamente, a um trabalho coletivo e cooperativo que se realizam entre sujeitos e se fazem numa rede de relações que exigem interação e diálogo. Estudos destacam a importância da contribuição decorrente das interações entre profissionais e usuários para o estabelecimento de relações de longa duração que promovam a efetividade da assistência prestada ${ }^{(7)}$.

O cuidar da família como conjunto único de saúde exige conhecê-la, identificar suas dificuldades e suas potencialidades. Só assim, o profissional, com seu saber técnico, científico e humanístico, poderá auxiliá-la a agir de forma a atender às necessidades de seus membros ${ }^{(5)}$. Para isso, é necessário haver máxima interação entre os profissionais de saúde e a família dos usuários assistidos nas equipes de saúde.

Assim, este estudo objetivou identificar o tipo de interação que os profissionais de saúde exercem com as famílias nas ESFs de um município do interior do Estado de Minas Gerais.

\section{MATERIAL E MÉTODOS}

Trata-se de uma pesquisa exploratória descritiva de abordagem qualitativa. O cenário do estudo foi constituído por cinco Unidades de Saúde da Família, 
localizadas na sede do município de Diamantina-MG.

A amostra foi composta por dez profissionais: médicos, enfermeiros e cirurgiões dentistas. A coleta de dados realizou-se no período de outubro a novembro de 2007, por meio de entrevistas semiestruturadas, com informações referentes a cada profissional sobre sexo, idade, categoria profissional, forma de contratação, tempo de formado e tempo em que trabalha na ESF. A entrevista incluiu também a questão norteadora do trabalho: Qual o tipo de interação exercida entre a sua equipe de saúde e a população da área de abrangência?

Todas as entrevistas aconteceram nos locais de trabalho dos profissionais, em sala e horários individuais, sendo gravadas em formato digital e posteriormente transcritas.

A análise dos dados fundamentou-se na proposta de interpretação qualitativa, modalidade temática baseada no método de análise de conteúdo ${ }^{(8)}$.

A pesquisa foi aprovada pelo Comitê de Ética e Pesquisa da Universidade Federal dos Vales do Jequitinhonha e Mucuri-UFVJM, respeitando as normas éticas em pesquisas com seres humanos da Resolução nº 196/96. Para cada sujeito da pesquisa, antes do início da coleta de dados, foi entregue e explicado para ser assinado, o Termo de Consentimento Livre e Esclarecido.

\section{RESULTADOS E DISCUSSÃO}

Os resultados evidenciam a proporção da amostra em relação à categoria profissional, sendo formada por 4 enfermeiros (40\%), 4 médicos (40\%) e 2 odontólogos (20\%). Constata-se também a predominância do sexo feminino (70\%). As faixas etárias dos entrevistados encontram-se entre 28 e 78 anos, com intervalos de 20 e 30 anos (30\%), 30 e 40 anos (20\%), 40 e 70 anos (30\%) e 70 anos ou mais (20\%). Em relação ao tempo decorrido após a formação acadêmica, há uma variação entre 5 e 10 anos (60\%), entre 11 e 20 anos (20\%) e 21 anos ou mais (20\%). Os profissionais em grande maioria foram admitidos no serviço por meio de processo seletivo simplificado (60\%) e (40\%) por contrato temporário. Sobre o tempo de atuação na ESF, houve variação de 1 mês a 5 anos (60\%) e de 6 a 10 anos (40\%).

Por meio das análises das respostas dos profissionais das equipes de saúde, pudemos construir unidades temáticas em um tema central: Atividades nas quais se realiza a interação profissional com as famílias atendidas pela Equipe de Saúde da Família. As unidades temáticas compreendidas desse tema foram: Visitas domiciliares, Consultas, Comunicação e Trabalho dos Agentes Comunitários de Saúde.

\section{Visitas Domiciliares}

A visita domiciliar foi a forma de interação que se destacou nos discursos analisados.

No que diz respeito à questão da saúde em cada casa que a gente visita, raramente a gente encontra resistência, mas a gente procura discutir, mostrar e acaba conseguindo bom resultado da visita que a gente faz a diversas famílias que são visitadas (Profissional 1).

[...] nas visitas você faz, você faz uma ligação das pessoas (Profissional 4).

[...] a gente tá muito ligado à família dos pacientes, com a sua família porque a gente tá sim, em contato na própria casa das pessoas e através das visitas domiciliares (Profissional 7).

Então a gente tenta trabalhar junto da família dentro da casa [...] (Profissional 9).

[...] toda segunda feira a gente faz oito visitas, vai [...] vai em oito famílias [...] (Profissional 10).

Quando nos aproximamos da realidade das pessoas, o que é proporcionado pelas visitas, abre-se caminho para uma melhor interação com a família em aspectos cada vez mais peculiares. Estudos destacam a presença de segredos de relacionamento ou de qualquer outra natureza dentro de um grupo familiar, afetando os processos de comunicação que precisam ser estabelecidos com o profissional ${ }^{(9)}$. A localização desses segredos molda as considerações clínicas e as possibilidades de intervenção na família. Portanto, as visitas domiciliares permitem uma relação mais estreita entre profissional e família, sendo um instrumento para conhecer a realidade das famílias atendidas.

\section{Consultas}

As relações da equipe com a comunidade estão diretamente ligadas à busca de consulta médica e medicamento. Apesar disso, há um reconhecimento 
do avanço nos aspectos preventivos.

Alguns autores contrastam atendimentos nos quais há uma abordagem mais cooperativa na comunicação com o paciente de uma consulta centralizada na passividade do indivíduo, quando há uma preocupação apenas com o seu estado físico ${ }^{(10)}$.

[...] porque através das consultas, você vai conhecendo a população, interagindo, identificando as necessidades e conflitos sociais [...] (Profissional 8).

[...] quando o paciente vem no meu consultório, a gente faz investigação, faz anamnese, eu procuro levar também aquela informação que o paciente me passou no consultório, no PSF, condiz com a realidade dele na família, se sim ou não (Profissional 5).

Notamos que as consultas são, para esses profissionais, formas de interação com a família, pois por meio delas, é colhida uma série de informações necessárias não somente ao exame clínico, mas também para inteirar-se sobre a situação social e uma possível orientação para cada conflito que a pessoa apresente.

\section{Comunicação}

O profissional muitas vezes atua como apaziguador de inquietações, que escuta, dialoga e orienta o paciente como lidar melhor com seus problemas.

Estudos apresentam que há um método suave de aconselhamento em conversas dos profissionais da saúde com pacientes. Os autores relacionam esse método com a prática cotidiana por meio dos estilos comuns de comunicação no tratamento de saúde, direcionamento, orientação e acompanhamento, nos quais abrigam habilidades como perguntar, escutar e informar ${ }^{(11)}$.

Os relatos dos profissionais refletem a comunicação como modo de interação com os indivíduos, bem como um envolvimento maior com os conflitos das famílias.

[...] de entender um pouquinho de conversar, escutar, pois um pouquinho de psicólogo mesmo, a gente é, do paciente (Profissional 3).

[...] mas a gente conversa, a gente tenta ver o que pode fazer para aquela pessoa. Começa a conversar, a ser amiga, tentar mostrar que a gente não é aquele bicho de sete cabeças [...] (Profissional 3).

[...] é um trabalho sensível, de escuta, comunicação [...] (Profissional 7).

Por meio dos relatos, pode-se perceber que os profissionais se relacionam de forma favorável, no sentido de conversar e entender o paciente. Isso se justifica porque todos precisam se comunicar, compartilhar sentimento, ideias, expectativas e situações $^{(12)}$.

\section{Trabalho dos agentes comunitários de saúde}

O trabalho do ACS está centrado no foco domiciliar e no acompanhamento das famílias cadastradas por sua equipe ${ }^{(13)}$. Deve-se ressaltar, assim, o importante elo entre os profissionais de saúde e as famílias, que são os Agentes Comunitários de Saúde.

\section{[...] através dos agentes comunitários (Profissional} 1).

[...] os agentes sempre nos trazem informações gerais que a gente acaba conhecendo a família (Profissional 4).

[...] por residirem na área os agentes nos ajudam a interagir com as famílias (Profissional 2).

A equipe troca informações com os ACS, pois cada profissional é chamado a desempenhar sua profissão em um processo de trabalho coletivo, cujo produto deve ser fruto de um trabalho que se forja com a contribuição específica das diversas áreas profissionais ou de conhecimento ${ }^{(14)}$.

As falas dos profissionais mostram um convívio satisfatório por meio da relação profissional/família, o que facilita a relação de confiança e respeito. Essa equipe e a população acompanhada criam vínculos de co-responsabilidade, o que facilita a identificação, o acolhimento, $\mathrm{o}$ atendimento e o acompanhamento dos agravos à saúde dos indivíduos e famílias na comunidade ${ }^{(15)}$.

\section{CONSIDERAÇÕES FINAIS}

Neste trabalho as formas de interagir com as famílias se pautaram em visitas domiciliares, 
comunicação, consultas e pelo trabalho do Agente Comunitário de Saúde.

Constatou-se que os profissionais de saúde têm a consciência de que é de fundamental importância a relação com as famílias atendidas, como forma empírica de conhecê-las e de alcançar convívio satisfatório de confiança e respeito, a fim de promover uma assistência à saúde de qualidade.

Espera-se com esse estudo que novas pesquisas sejam desenvolvidas, uma vez que a ESF tem sido considerada como uma forma de reorganização da ABS e, para que isso realmente aconteça e se efetive, os profissionais necessitam interagir com as famílias por eles assistidas.

\section{REFERÊNCIAS}

1. Szymanski H. Viver em família como experiência de cuidado mútuo: desafios de um mundo em mudança. Serviço Social e Sociedade. 2002; ano XXII.

2. Azevedo MRD. Educação sexual: uma questão em aberto. In: Saito MI, Silva LEV, organizadores. Adolescência: prevenção e risco. São Paulo: Atheneu; 2001.p.129-43.

3. Ribeiro LCC, Ribeiro M, Dias KS, Matos KA, Ferreira TS. O diagnóstico administrativo e situacional como instrumento para o planejamento de ações na Estratégia Saúde da Família. Cogitare Enferm. 2008 Jul/ Set;13(3):448-52.

4. Marcon SS, Elsen I. A enfermagem com um novo olhar... a necessidade de enxergar a família. Fam Saúde Desenv. 1999 Jan/Dez;1(1/2):21-26.

5. Oliveira RG, Marcon SS. Trabalhar com famílias no Programa de Saúde da Família: a prática do enfermeiro em Maringá-Paraná. Rev Esc Enferm USP. 2007;41(1):6572.

6. Ministério da Saúde (BR). Departamento de Atenção Básica. Guia prático do programa de saúde da família. Brasília, Ministério da Saúde, 2001; 128 p.

7. Starfield B. Atenção primária: equilíbrio entre necessidades de saúde, serviços e tecnologia. Brasília: UNESCO; Ministério da Saúde; 2002.

8. Bardin L. Análise de conteúdo. Lisboa: Edições 70; 1977.

9. Imber-black E. Os segredos na família e na terapia familiar. Porto Alegre: Artmed; 1994.

10. Miller WR, Rollnick S. Entrevista motivacional: preparando as pessoas para a mudança de comportamentos adictivos. Porto Alegre, RS: Artmed; 2001.

11. Bonfim LA, Bastos ACS. O impacto sócio-cultural do Programa de Saúde da Família (PSF): uma proposta de avaliação. Cad Saúde Publ. 1998;14(2): 429-35.

12. Pedrosa JTS, Teles JBM. Consenso e diferenças em equipes do Programa Saúde da Família. Rev Saúde Publ. 2001;35:303-1.

13. Costa GP. Conflitos da vida real. $2^{\mathrm{a}}$ ed., rev. e ampl. Porto Alegre: Artmed; 2006.

14. Silva IZQJ, Trad LAB. O trabalho em equipe no PSF: investigando a articulação técnica e a interação entre os profissionais. Interface - Comunicação, Saúde e Educação. 2005; 9(16):25-38.

15. Monteiro MM, Figueiredo VP, Machado MFAS. Formação do vínculo na implantação do Programa Saúde da Família numa Unidade Básica de Saúde. Rev Esc Enferm USP. 2009;43(2):358-64. 